\title{
Alterstice
}

Revue internationale de la recherche interculturelle

International Journal of Intercultural Research

Revista International de la Investigacion Intercultural

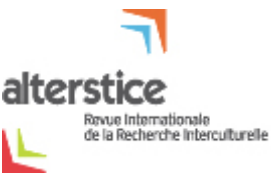

\section{Le père japonais dans une société ultramobile de célibataires}

\section{Valérie Harvey et Diane-Gabrielle Tremblay}

Volume 5, numéro 1, 2015

Engagement paternel et migration

URI : https://id.erudit.org/iderudit/1077304ar

DOI : https://doi.org/10.7202/1077304ar

Aller au sommaire du numéro

Éditeur(s)

Alterstice

ISSN

1923-919X (numérique)

Découvrir la revue

Citer cet article

Harvey, V. \& Tremblay, D.-G. (2015). Le père japonais dans une société

ultramobile de célibataires. Alterstice, 5(1), 7-21.

https://doi.org/10.7202/1077304ar
Résumé de l'article

Selon Esping-Andersen, les politiques publiques adoptées par les pays se classent selon trois grands modèles de relation travail-famille et le Japon s'inscrit dans le contexte non interventionniste. La théorie de la modernité avancée développée par Beck divise les possibilités d'organisation du travail en trois types pour les familles. En observant attentivement le peu de mesures mises en place par l'État japonais pour encourager la parentalité, l'absence de soutien envers les pères japonais contribue à faire du Japon une société ultramobile de célibataires, ou du moins à se rapprocher fortement de cette vision. Notre texte soutient cette hypothèse en traitant des congés parentaux et de la division du travail dans un ménage japonais et des effets de l'ensemble sur la famille au Japon. 


\section{7}

alterstice

Revue Internationale

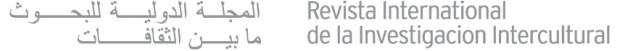

ARTICLE THÉMATHIQUE

\section{Le père japonais dans une société ultramobile de célibataires}

Valérie Harvey ${ }^{1}$ et Diane-Gabrielle Tremblay ${ }^{1,2}$

\section{Résumé}

Selon Esping-Andersen, les politiques publiques adoptées par les pays se classent selon trois grands modèles de relation travail-famille et le Japon s'inscrit dans le contexte non interventionniste. La théorie de la modernité avancée développée par Beck divise les possibilités d'organisation du travail en trois types pour les familles. En observant attentivement le peu de mesures mises en place par l'État japonais pour encourager la parentalité, l'absence de soutien envers les pères japonais contribue à faire du Japon une société ultramobile de célibataires, ou du moins à se rapprocher fortement de cette vision. Notre texte soutient cette hypothèse en traitant des congés parentaux et de la division du travail dans un ménage japonais et des effets de l'ensemble sur la famille au Japon.

\section{Rattachement des auteures}

${ }^{1}$ Alliance de recherche université communauté (ARUC) sur la gestion des âges et des temps sociaux (GATS), Montréal, Canada

${ }^{2}$ TELUQ

\section{Correspondance}

vh.sociologie@gmail.com

\section{Mots clés}

Japon, congé parental, congé paternel, pères, mères, égalité, enfant, politiques familiales

\section{Pour citer cet article}

Harvey, V. et Tremblay, D.-G. (2015). Le père japonais dans une société ultramobile de célibataires. Alterstice, 5(1), 7-22. 


\section{男は仕事、女は家庭。}

(Les hommes ont le travail, les femmes ont la maison)

Proverbe japonais

Dans le touchant film réalisé par Hirokazu Kore-eda, Tel père, tel fils (そして父になる), un couple aisé apprend que leur fils de six ans a été échangé à la naissance avec un autre venant d'une famille plus modeste. Le mari architecte reproche alors à sa femme d'avoir choisi d'accoucher dans cet hôpital de campagne reculée. Elle lui réplique qu'il travaillait tout le temps et qu'elle a rejoint sa mère pour ne pas vivre l'accouchement et ses suites toute seule. Ce faisant, elle exprime la réalité d'un grand nombre de mères japonaises, qui vivent la parentalité dans une grande solitude. Paradoxalement, Holloway mentionnait qu'au Japon "Most of the women did not expect their husbands to participate extensively in housework, but they did want them to be actively involved with their children. The women also wanted their husbands to provide emotional support, mostly by listening carefully and sympathetically to their worries " (Holloway, 2010, p. 203).

Qu'en est-il réellement? Nous présenterons d'abord le modèle non interventionniste de l'État japonais à partir d'une recherche documentaire. Quels ont été les résultats de ces politiques familiales? Est-ce que ces initiatives ont porté les pères à s'impliquer davantage par la suite? Nous tenterons ensuite d'expliquer l'échec des mesures mises en place par la théorie de la société du risque développée par Ulrich Beck, en faisant d'abord un rappel des éléments pertinents de la théorie de Beck puis en exposant les trois grands modèles de relation travail-famille afin de situer le Japon par rapport au Québec.

Cette recherche présente bien sûr des limites, puisqu'elle est fondée sur une revue des écrits et non sur une enquête directe au Japon. Cependant, une des auteures parlant le japonais et ayant séjourné un peu plus d'un an dans le pays, des travaux et des sources relativement inconnus hors du Japon ont pu être exploités. Des recherches pourront être menées ultérieurement au Japon, auprès des acteurs sociaux (comme les syndicats) susceptibles d'avoir des opinions sur ces politiques, ou grâce à des analyses plus approfondies de situations d'hommes et de femmes. Cet article propose pour l'instant une interprétation globale sur les pères et la famille japonaise, permettant une première approche du sujet.

\section{La théorie de Beck}

Le sociologue Ulrich Bech définit la famille de la modernité simple, soit celle des années 1950, comme une institution basée sur une division des rôles en fonction du sexe. Il accorde beaucoup d'importance à cette répartition des tâches en arguant qu'elle est nécessaire au fonctionnement même de la société industrielle : "La répartition des rôles en fonction de l'appartenance sexuelle est la base de la société industrielle [...] La société industrielle est donc dépendante des situations inégales des hommes et des femmes. » (Beck, 2001, p. 235).

Cette différenciation sexuée, que Parsons (1964) avait jugé nécessaire pour que le père et la mère assument leurs fonctions différentes et complémentaires, est portée par la même modernité qui prône la liberté individuelle, l'égalité des sexes, l'importance d'une bonne éducation dans un marché qui accordera de plus en plus de place au savoir et enfin l'autonomie que permet un emploi. II y a clairement des « [...] oppositions entre le capital et travail, le produit et le fondement du système industriel, et ce dans la mesure où l'activité professionnelle présuppose l'existence du travail domestique » (Beck, 2001, p. 239).

Malgré le fait que les femmes aient maintenant accès à la même éducation et qu'elles soient présentes sur le marché du travail, ce sont toujours elles qui assument la plus grande part des tâches ménagères et des soins aux enfants et, souvent, elles renoncent à une part de leurs revenus pour réussir à conjuguer travail et famille, ce qui peut signifier un appauvrissement lors d'un divorce ou de la retraite. C'est ainsi que « la famille devient le lieu où l'on jongle continuellement, avec de multiples ambitions contradictoires, entre les professions et les impératifs de mobilité qui en découlent, les contraintes imposées par la formation, les obligations liées aux enfants et au travail domestique »(Beck, 2001, p. 248). 
Pour Beck, la famille est le reflet de cette contradiction, car elle subit les effets pervers d'une modernité réflexive qui ne permet pas d'exercer pleinement les principes de liberté et d'égalité qu'elle prône (Beck, 2001, p. 240). La différenciation de la société moderne a ainsi créé des catégories fonctionnelles qui ont donné une impression de contrôle et de sécurité, sentiments aujourd'hui disparus dans la modernité avancée, véritable société du risque (Beck et Lau, 2005, p. 526).

La formation d'un couple n'est plus basée sur le respect des traditions ou sur une obligation. L'amour est évoqué comme base du couple actuel, mais « le couple et la famille tiennent moins par le fondement matériel et l'amour que par la peur de la solitude " (Beck, 2001, p. 253). Le nombre de personnes vivant seules augmente sans cesse et, pour Beck, cela n'est pas surprenant puisque la société mondialisée favorise l'individu seul. La famille et les couples sont devenus l'anachronisme :

Le marché du travail exige la mobilité sans tenir compte des situations personnelles. Le couple et la famille exigent le contraire. Dans le modèle de marché poussé à son paroxysme qui est caractéristique de la modernité, on présuppose que la société est exempte de familles et de couples. [...] Le sujet du marché est l'individu seul, débarrassé de tout « handicap » relationnel, conjugal ou familial. (Beck, 2001, p. 257)

Cette réalité d'une modernité avancée qui exige des individus libres, disponibles et mobiles crée d’immenses tensions entre les membres d'une famille, qui tentent tant bien que mal de les gérer de l'intérieur: " [...] on y voit surgir les conflits du siècle. Ils y apparaissent toujours sous un jour privé, familier. Mais la famille n'est que le lieu, elle n'est pas la cause de ce qui se produit "(Beck, 2001, p. 237). Le niveau de conflit augmente entre deux individus placés devant l'impossible tâche d'être autonomes et pourtant unis devant la responsabilité familiale, où l'enfant est devenu un obstacle au processus d'individualisation (Beck, 2001, p. 260) car « il oblige ses parents, avec force hurlements et éclats de rire, à adopter son rythme de vie de créature humaine. Mais d’un autre côté, c'est justement ce qui le rend irremplaçable. L'enfant devient le dernier lien primaire subsistant, irrévocable, résolument non interchangeable » (Beck, 2001, p. 260). Ces propos rejoignent Kaufmann qui, dans L'étrange histoire de l'amour heureux, décrit le couple amoureux attaqué de toute part par la marchandisation d'une société dominée par I'homo œconomicus, aux calculs de laquelle seul l'enfant échappe: "Dans notre société où tout se discute et se critique (même le conjoint donc), il est la seule évidence, absolue; qui donne un nouveau sens, plein et entier, à la vie " (Kaufmann, 2009, p. 184). Mais si l'enfant est tout-puissant et bénéficie d'un amour qui ne s'éteindra pas avec le temps, le bébé n'est pas sans conséquence pour l’homme et la femme, étant donné les rôles encore attribués à l'un ou l'autre des sexes: "Le moment que l'on choisit pour faire un enfant représente des conditions et des obstacles très différents dans l'existence masculine et féminine » (Beck, 2001, p. 259). La tension ainsi créée explique, pour Beck, la diminution des naissances et l'augmentation de la courbe des divorces (Beck, 2001, p. 259).

Face à cette contradiction, trois possibilités d'avenir existent selon Beck, et la société doit répondre simultanément à ces défis (Beck et Lau, 2005, p. 526). Elles coexistent souvent en une seule et même société.

D’abord, il y a la possibilité du retour à la famille dans ses formes traditionnelles, ce qui évidemment permet d'éviter les tensions de conciliation travail-famille. La femme restant à la maison, la partie "travail » de la conciliation exigée est donc retirée. C'est une alternative valable, particulièrement pour celles qui n'ont pas une vie professionnelle particulièrement intéressante ou suffisante pour être autonomes. Mais il reste que ces femmes se retrouvent souvent démunies lors d'une séparation. De plus, une application globale de cette solution est impossible puisque plusieurs femmes ne désirent pas ce style de vie. L'appliquer à toutes les familles équivaudrait donc à augmenter les frustrations, ce qui « [...] aboutira à un renforcement des conflits relationnels induits par des causes extérieures " (Beck, 2001, p. 265). Cette solution existe certainement au Japon puisque les emplois des femmes entre 20 et 30 ans sont, pour une large part, des postes de OL, soit office lady : elles effectuent des tâches simples et répétitives qui aident les employés réguliers (majoritairement masculins) à remplir leur mandat : "Les OL s'occupent de tâches peu spécialisées, comme classer des dossiers, faire des photocopies ou servir le thé [...] " (Bernier, 2009, p. 139). Ce n'est, bien entendu, pas un poste où l'on fait une longue carrière puisque l'employeur s'attend généralement à ce que l'office lady quitte cet emploi à son mariage ou à la venue du premier enfant. Cette façon de faire est encore la norme au Japon, ce pays étant l'un des seuls parmi les 30 démocraties étudiées par l'OCDE qui présente toujours, en 2005, une courbe d'évolution du taux d'emploi selon l'âge en forme de M pour les 
femmes (OCDE, 2007, p. 8). Cela signifie que, même si le taux d'emploi des femmes de 20 à 30 ans est élevé et ne cesse d'augmenter, se situant autour de $70 \%$ en 2007 (Keizai Koho Center, 2010, p. 83), $40 \%$ des femmes se retirent encore à la naissance du premier enfant, puis retournent sur le marché du travail entre 40 et 50 ans (Bernier, 2009, p. 183). Seulement $22 \%$ des mères ayant un enfant d'âge préscolaire continuent d'exercer l'emploi qu'elles occupaient avant sa venue au monde (Holloway, 2010, p. 172).

Lorsque ces mères retournent sur le marché du travail ou lorsqu'elles décident de travailler en même temps qu'elles prennent soin de leur jeune enfant, elles choisissent (ou n'ont souvent d'autre choix) d'occuper un emploi à temps partiel pour réussir à concilier travail et famille. Il est difficile de revenir dans le parcours régulier après un arrêt de travail, puisque les entreprises privilégient les nouveaux diplômés, qui sont plus faciles à modeler pour répondre à leurs normes. Un sondage gouvernemental a révélé en 2001 que, malgré des lois interdisant la discrimination fondée sur l'âge, près de $90 \%$ des entreprises établissaient un âge limite d'embauche (Holloway, 2010, p. 177).

Ensuite, Beck évoque la possibilité de créer une "société ultramobile de célibataires ", où l'on dissout carrément la famille et qui tourne autour du moi central, ce qui « [...] présente le risque de constituer un obstacle insurmontable à la constitution d'une relation (couple, famille) qui pourtant est la plupart du temps désirée » (Beck, 2001, p. 268). C'est donc dire ici que l'on supprime la partie "famille » de la fameuse conciliation. Au Japon, le mariage est essentiel à la venue d'un enfant. Les statistiques sont formelles : le nombre de naissances hors mariage y est quasi inexistant, le pourcentage d’enfants nés hors mariage étant évalué à 1,9\% de toutes les naissances en 2002 (Rebick, 2006, p. 8). Au vu du nombre de mariages qui ne cesse de diminuer au pays du soleil levant, le Japon pourrait donc bien être un exemple de société ultramobile de célibataires. Ainsi, l'âge moyen au moment du mariage ne cesse de s'élever. Plus menaçant encore est le nombre croissant de Japonaises non mariées : il a triplé entre 1970 et 2005. Les chercheurs estiment que plus de $20 \%$ des Japonaises de la génération montante (entre 25 et 34 ans) ne se marieront pas (Bumpass, Rinfuss, Choe et Tsuya, 2009, p. 218), 12 \% se marieront mais n'auront pas d'enfant, tandis que $16 \%$ auront un seul enfant. Ce qui permet de déduire que près du tiers (32 \%) des femmes resteront sans enfant. Si on inclut celles qui auront un enfant unique, ce sont alors près de de la moitié (48 \%) des femmes qui auront un seul enfant ou aucun enfant (Retherford et Ogawa, 2005, p. 4).

Dans ce type de société, la pression sociale est forte pour que même les couples qui ont des enfants se soumettent aux exigences de l'entreprise. Un sondage révèle que les pères souhaitant s'impliquer auprès de leur famille se sont retrouvés face à des obstacles inattendus: «11\% des hommes interrogés avouent avoir été victimes de harcèlement en raison de leur attention paternelle, et $5 \%$ ont vu leurs demandes de congés paternité refusées. $3,8 \%$ ont entendu leur patron leur dire que s'ils prenaient ce congé, leur carrière en pâtirait. 10,8 \% admettent avoir assisté à la souffrance de leurs collègues masculins, en raison de leur paternité, et de leur désir d'y accorder du temps ", selon Rengo, la Confédération des syndicats japonais (Braibant, 2014).

Citons également le tanshin funin (単身赴任), cette pratique qu'ont les entreprises de transférer un homme dans une autre ville japonaise, alors que sa femme et ses enfants restent dans la ville d'origine pour toutes sortes de raisons: l'enfant a passé les examens d'entrée d'une école huppée, la femme a ses amies dans cet endroit, la maison est acquise, etc. Le père rend visite à sa famille environ une fois par mois, et devient pratiquement un étranger pour ses enfants. À ce niveau, la langue japonaise est révélatrice du mode de vie imposé aux travailleurs : on peut décrire ce phénomène en combinant le nom de la ville avec " chonga ", un terme signifiant " célibataire " en coréen. Ainsi, le père muté à Sapporo sera appelé un "Sacchon ». Cela vient rejoindre notre hypothèse formulant que le Japon est un bon exemple de société ultramobile de célibataires, même si les célibataires en question n'ont plus ce statut.

Beck propose une solution alternative, qui n'est pas nécessairement simple. II la présente comme l'expérimentation de nouveaux modes de vie qui transcendent les rôles des hommes et des femmes :

L'égalité entre hommes et femmes ne peut se réaliser dans des structures institutionnelles qui reposent justement sur l'inégalité. Ce n'est qu'en repensant et en modifiant tout l'édifice institutionnel de la société industrielle développée, en mettant en place les conditions nécessaires à la vie de famille et à la vie de couple que l'on pourra atteindre peu à peu une 
égalité d'un genre nouveau, qui se jouera au-delà de la répartition traditionnelle des rôles masculins et féminins. (Beck, 2001, p. 270)

Pour ce faire, les solutions devront venir de l'État, qui doit cesser d'être en décalage institutionnel, ce qui devrait permettre d'augmenter les possibilités pour les couples. Cependant, les modèles ne viendront pas du haut, " [les nouveaux collectifs] doivent être inventés, construits, élaborés, négociés par le bas » (Beck, 1998, p. 20). L’État doit plutôt s'assurer de mettre en place des politiques publiques diversifiées et flexibles qui permettent aux couples de trouver, d'inventer et d'expérimenter d'autres formes de vie commune qui ne se calquent pas sur une répartition traditionnelle des rôles (Beck, 2001, p. 273). Le but n'est pas de transférer les charges domestiques sur le dos des hommes pour permettre aux femmes de travailler, mais de changer le système moderne avancé qui bloque les couples et les familles, en plus d'être responsable de la majeure partie des tensions présentes dans les familles d'aujourd'hui. Dans cette voie de solution, on pourrait probablement classer les mesures les plus populaires des pays nordiques et du Québec, notamment les congés parentaux et les services de garde, qui facilitent la conciliation. Mais voyons les différents modèles de relation travail-famille, pour mieux classer le Japon dans ce cadre.

\section{Les trois grands modèles de relation travail-famille}

Les recherches sur les modèles de conciliation travail-famille ont permis d'identifier trois grands modèles d'adaptation. Ces modèles varient selon les pays et les régions géographiques. L'Europe du Nord et du Sud s'opposent clairement, tout comme l'Amérique du Nord (les États-Unis surtout) et l'Europe. Cette diversité montre que la relation emploi-famille est une réalité socialement construite. L'articulation emploi-famille prend des formes très différentes selon le contexte social, démographique, culturel, mais aussi - et presque surtout, diraient certains - selon les politiques publiques en place.

Pour saisir la diversité des situations et pour comprendre leurs logiques particulières, nous avons procédé à une comparaison internationale des diverses politiques. On constate qu'il se dégage assez clairement des types ou des modèles caractéristiques des modes d'adaptation de la relation emploi-famille, même si aucun de ces modèles ne se retrouve à l'état pur dans la réalité. En pratique, nombre de pays constituent des cas hybrides qui regroupent des composantes appartenant aux trois modèles. La plupart des pays se rapprochent davantage de tel ou tel modèle, mais sans jamais y correspondre tout à fait. Les modèles sont plutôt des types idéaux qui permettent de classer les pays tantôt en fonction des aspects par lesquels ils s'apparentent, tantôt en fonction des aspects par lesquels ils se distinguent. Nous retenons donc comme base les trois grands modèles institutionnels identifiés entre autres par Hantrais et Letablier $(1995,1996)$. Nous présentons quelques éléments de ces modèles avant de passer à l'analyse de la situation japonaise.

- Le modèle de la conciliation entre emploi et famille. Dans les pays s'approchant de ce modèle, l'intervention publique a pour objectif de concilier les exigences de la vie familiale et celles de l'emploi, en permettant aux individus, femmes et hommes, de se maintenir en emploi tout en assumant leurs responsabilités familiales. Autrement dit, la conciliation permet de juxtaposer la famille et l'emploi sans avoir à sacrifier l'un ou l'autre ${ }^{1}$.

- Le modèle de l'alternance entre emploi et famille. Par rapport au modèle de la conciliation, le modèle de l'alternance n'a pas pour objectif prioritaire de juxtaposer la famille et l'emploi ou, en d'autres termes, de permettre de se consacrer simultanément aux deux activités. Dans les pays orientés vers ce modèle, l'intervention publique incite plutôt les parents en emploi, généralement les femmes, à opter pour une stratégie d'entrée et de sortie du marché du travail afin de " concilier » emploi et famille, mais en priorisant l'un et l'autre à des moments différents. Autrement dit, l'État encourage les femmes à quitter leur emploi ou à réduire leur temps de travail pour prendre soin de leurs enfants, puis à revenir plus tard sur le marché du travail, le plus souvent à temps partiel, lorsque les enfants atteignent l'âge scolaire. À nos yeux, il ne s'agit plus à proprement parler de conciliation dans ce cas, puisque cette notion suppose que les parents - ou les mères en particulier - parviennent à faire les deux choses en même temps, se maintenir en emploi et assumer

\footnotetext{
${ }^{1}$ Voir les articles sur divers pays dans Moss (2010), ainsi que Moss et Kamerman (2009) pour l'historique du développement des congés parentaux dans divers pays.
} 
leurs responsabilités familiales, sans devoir délaisser l'une ou l'autre. À noter que nous parlons surtout des mères puisque même si l'on peut souhaiter que le partage soit plus équitable entre les parents, la famille pose rarement un véritable problème de conciliation pour le père et rares sont ceux qui sont obligés d'abandonner leur travail pour se consacrer à leur famille. Ce sont plus souvent les femmes qui font ces ajustements et elles n'obtiennent pas toujours le soutien nécessaire de la part de leur employeur.

- Le modèle non interventionniste. Les pays orientés vers ce modèle se caractérisent par une quasi-absence de mesures étatiques en matière d'ajustement de la relation emploi-famille. Parmi ces pays, il convient de distinguer ceux où l'État intervient peu ou pas en raison d'une insuffisance de ressources, comme certains pays de l'Europe du Sud, et ceux où la faiblesse de l'intervention étatique s'explique d'abord par des raisons de principe, comme au Royaume-Uni ou aux États-Unis. Dans ce dernier cas, l'État et ses interventions, conformément à la tradition philosophique du libéralisme qui prédomine dans les pays anglo-saxons, sont considérés comme une menace potentielle à la liberté de l'individu ${ }^{2}$. II en découle une conception purement privatiste de l'articulation emploi-famille, laissée tout entière à l'initiative des individus et des employeurs. On peut conclure que, dans ce dernier cas, les négociations collectives à l'échelle de l'entreprise doivent suppléer à l'absence de politique publique et d'intervention étatique, mais il est possible que cela ne se produise pas si la société semble à l'aise avec une forte division du travail entre hommes et femmes et avec le maintien d'un modèle traditionnel à un seul pourvoyeur de revenus, soit l'homme.

Passons maintenant à l'analyse de la situation des pères au Japon, qui permet de confirmer que le pays suit le troisième modèle, celui des pays non interventionnistes (Powell et Barrientos, 2004, p. 94), et qu'il se rapproche de la définition d'une société ultramobile de célibataires, selon la théorie de Beck précédemment exposée.

\section{La situation des pères au Japon}

Le plus haut bureau du gouvernement japonais, le Cabinet Office, a publié en 2001 un rapport qui compare la situation des hommes et des femmes ayant de jeunes enfants et travaillant à temps plein. Les hommes s'adonnent aux tâches ménagères un court cinq minutes par jour, alors qu'une femme dans la même situation y passe trois heures et demie. Dans la population générale, les femmes japonaises consacrent presque 30 heures par semaine aux tâches ménagères, contre une moyenne de 2,5 heures pour les hommes, ce qui inclut un $30 \%$ d'hommes qui n'en font aucune (Bumpass, Rinfuss, Choe et Tsuya, 2009, p. 221). Les pères ne consacrent qu'une demi-heure au soin des enfants, contre quatre heures pour la mère travaillant à temps plein (Nakatani, 2006, p. 8) (figure 1).

Ces statistiques confirment que les femmes japonaises travaillant à temps plein sont encore les principales responsables de la gestion de la résidence et de la famille. Elles doivent réussir à accomplir leurs tâches domestiques et familiales tout en occupant un emploi et en récupérant à l'heure leur enfant à la garderie. Ce partage inégal des tâches rend la conciliation travail-famille extrêmement difficile, et fort peu de femmes peuvent (et veulent) reprendre un travail régulier après l'arrivée d'un enfant (Bumpass, Rinfuss, Choe et Tsuya, 2009, p. 220; Holloway, 2010, p. 178; Jolivet, 1993, p. 63; OCDE, 2007, p. 40) : "Celles qui exercent un métier qu'elles veulent conserver (médecin, infirmière, enseignante, etc.) ont un maintien au travail plus élevé. Néanmoins, une bonne partie des femmes qui continuent de travailler le font à temps partiel »(Bernier, 2009, p. 184).

La charge de travail d'une femme occupant un emploi de 42 heures ou plus par semaine à laquelle s'ajoutent les tâches domestiques est de 73 heures par semaine, et cela avant même que le soin des enfants soit ajouté à ces données. Finalement, les statistiques indiquent qu'une femme qui travaille réduira d'environ huit heures le temps consacré aux tâches ménagères lorsqu'elle aura un enfant, mais que la plupart des maris n'augmenteront pas leur contribution aux tâches domestiques (Bumpass, Rinfuss, Choe et Tsuya, 2009, p. 222).

${ }^{2}$ Voir à ce sujet Lesemann et Nicol (1994). 


\section{Figure 1 Nombre d'heures par jour consacré par les pères aux tâches domestiques et au soin des enfants (2009)}

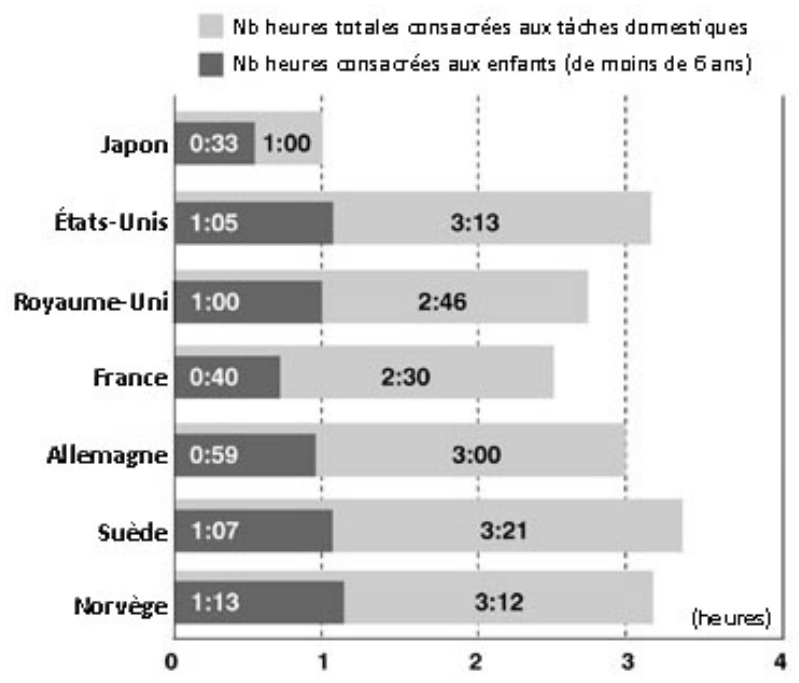

Source : Eurostat, Bureau of Labor Statistics of the U.S., Ministère des Affaires internes et des Communications (Japon)

Non seulement il est presque impossible de concilier un travail à temps plein et le soin d'une famille, mais, financièrement, il n'est pas avantageux d'avoir deux personnes occupant un emploi régulier au sein du ménage. Les épouses n'ont pas à payer de cotisations de sécurité sociale sur un revenu personnel annuel atteignant 1,03 million de yens, soit environ 10000 dollars canadiens (Hirayama et Izuhara, 2008, p. 647; OCDE, 2007, p. 208). Le fait que l'État considère toujours la famille comme une unité et impose des pénalités aux familles à deux revenus décourage la femme qui a un revenu moyen, car elle peut bénéficier de meilleures conditions en arrêtant tout simplement de travailler (Holloway, 2010, p. 196).

Une des conséquences est visible: la natalité et la population s'en ressentent, le Japan Center for Economic Research estimant que la population active japonaise passera à 44,5 millions de personnes en 2050, soit une baisse de $70 \%$ par rapport à 2005 (Holloway, 2010, p. 3). Dans un livre intitulé Shoushi (ce qui signifie littéralement « Peu d'enfants "), l'auteure Junko Sakai va encore plus loin dans les prédictions à la baisse puisqu'elle présente un graphique (figure 2) où elle estime qu'il ne restera que 500 Japonais en 2350 si la dénatalité continue à un tel rythme (Sakai, 2003, p. 19).

C'est au Japon qu'on observe l'un des indices de fécondité les plus bas au monde, c'est-à-dire 1,26 enfant par femme en 2005, alors que la moyenne des 30 pays de OCDE était de 1,63 enfant pour la même année (OCDE, 2007, p. 17). Les plus récentes études ont confirmé que la population masculine japonaise a atteint son sommet en mars 2005 et qu'elle décline depuis, l'espérance de vie des hommes étant moins élevée d'environ six ans que celles des femmes (Matsukura, Ogawa et Clark, 2007, p. 84). En 2011, la population féminine a atteint son maximum et a commencé, elle aussi, à décroître. Le Japon a donc entamé le déclin de sa population totale.

Pourtant, plus le père consacre de temps au soin des enfants et de la maison, plus grandes sont les chances que le couple ait d'autres enfants (Ministry of Health, Labour and Welfare of Japan, 2009, p. 20). Il est également prouvé qu'une jeune femme célibataire ayant eu un père impliqué dans les tâches familiales et domestiques manifestera plus tôt le désir de se marier et d'avoir des enfants, parce qu'elle aura une meilleure opinion du mariage (Retherford, Ogawa et Matsukura, 2001, p. 93). Au contraire, une femme ayant eu pour exemple un père peu impliqué hésitera plus longtemps à accepter "l'ensemble-mariage ", c'est-à-dire le sacrifice de sa carrière et le soin au mari et aux enfants, en plus du possible soutien aux parents âgés (Bumpass, Rinfuss, Choe et Tsuya, 2009, p. 218; Jolivet, 1993, p. 221). 
Figure 2 - Évolution de la population japonaise, d'après les données de 2001

\section{仮に、出生率、死亡率が2001年(平成13年)のままとした場合の 将来の日本の人口}

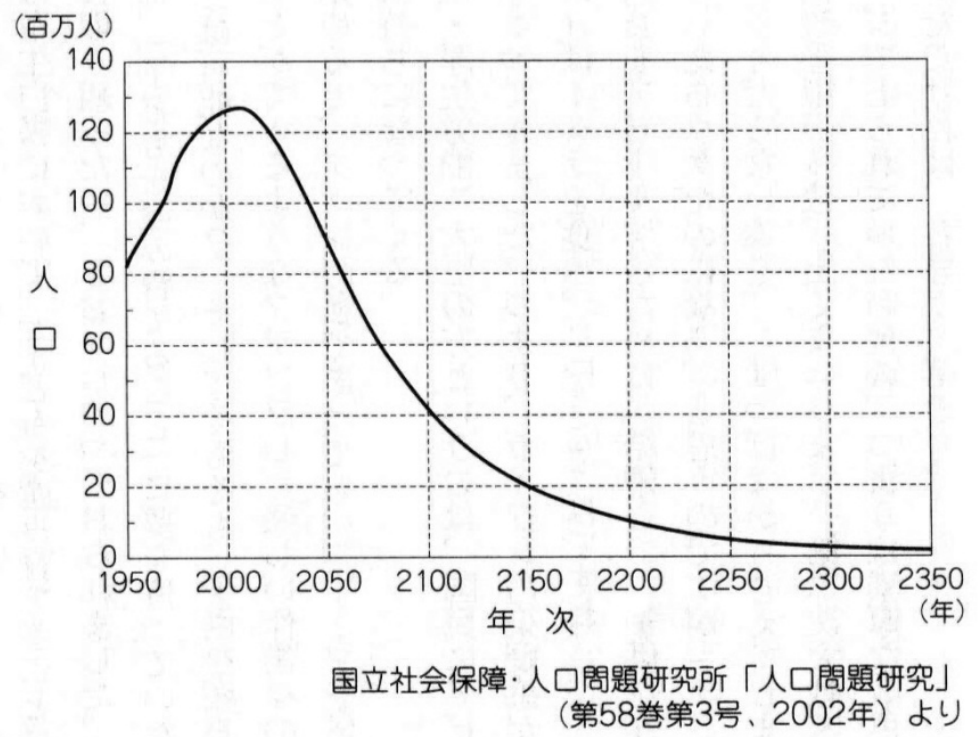

長生きして“人口500人” の状態を体験してみたい。

Source : Sakai (2009, p. 19)

\section{Les congés parentaux japonais}

En 1979, alors que la Chine instaurait ses premières mesures restreignant le nombre de naissances, les autorités japonaises songèrent plutôt à appuyer la fécondité par des mesures natalistes. On commença à parler de retrait pour le congé maternel et à former des comités pour instaurer un plan d'action. Pour la première fois, on évoqua le rôle du père et l'on pointa du doigt la baisse du nombre de mariages.

En 1991, en parallèle avec la Loi sur l'égalité des chances mise en place en 1985 pour assurer aux femmes de meilleures opportunités sur le marché du travail, le gouvernement met en place le Childcare Leave Act qui permett aux employées de type régulier (à temps plein) des entreprises de plus de 30 salariés de bénéficier d'un congé maternel, sans solde, d'une durée maximale d'une année. En 1995, le gouvernement remplace cette loi par le Childcare and Family Leave Act, incarné par l'Angel Plan. Ce plan couvre alors les entreprises de moins de 30 employés et fournit $25 \%$ du salaire à la mère en congé. L'entreprise doit également continuer de contribuer aux cotisations sociales d'une personne en congé maternel. En 2000, l'État reprend cette responsabilité pour réduire les coûts aux entreprises. En 2001, on améliore le régime en fournissant alors $40 \%$ du salaire et en ajoutant un congé de trois mois, sans solde, pour prendre soin d'un enfant ou d'une personne malade dans la famille. En 2005, une nouvelle révision du plan inclut les personnes travaillant à temps partiel. Finalement, en 2009, on permet aux personnes ayant de jeunes enfants de travailler seulement six heures par jour, sans faire d'heures supplémentaires. La nouvelle loi de 2009 permet à l'un des deux parents de prendre un congé parental sans solde jusqu'à ce que l'enfant ait 14 mois (au lieu de 12 mois précédemment).

Les mesures en place depuis 2001 ont eu des effets négatifs pour les femmes : elles ont donné lieu à de la discrimination. Les statistiques indiquent que les entreprises engagent moins de femmes pour des postes réguliers, craignant sans doute de les voir quitter leur emploi pour un congé maternel (Retherford et Ogawa, 2005, p. 31).

Dans un pays où les employés disposent en moyenne de 18 jours de congé annuellement, dont seulement $48 \%$ sont utilisés (OCDE, 2007, p. 211), le congé paternel ne fait pas exception à la règle. Un maigre $1,56 \%$ des pères 
l'avait utilisé en 2009 (Ministry of Health, Labour and Welfare of Japan, 2009, p. 28) : « Tant que les droits aux congés seront utilisés en grande majorité par les mères, plutôt que les pères, il y aura des employeurs qui considéreront que les femmes s'impliquent moins dans leur travail que les hommes [...] » (OCDE, 2007, p. 23-24).

De nombreuses recherches menées en Europe et en Asie ont démontré que les politiques publiques de soutien à la parentalité qui comprennent des mesures financières et des services de garde ont tendance à favoriser la fécondité (Holloway, 2010, p. 215). Au Canada,

les constatations découlant de l'Enquête sociale générale de 2006 démontrent que les personnes en couple qui veulent avoir des enfants sont beaucoup plus susceptibles de considérer l'accès à des prestations parentales comme "très important" dans leurs décisions en matière de fécondité si elles ont déjà un enfant, si elles font partie d'un couple où les deux conjoints travaillent, et si elles ont un revenu du ménage inférieur à $100000 \$$ par an. (Crompton et Keown, 2009, p. 54)

Quelques auteurs se sont penchés sur la question au Japon et en sont venus à la même conclusion : les femmes ayant la possibilité de prendre un retrait parental sans que leur emploi ne soit menacé ont plus de chances d'avoir un enfant (Ministry of Health, Labour and Welfare of Japan, 2009, p. 6) et cela augmente également la possibilité d'avoir un deuxième enfant (Lee, Ogawa et Matsukura, 2009, p. 364).

Plusieurs études ont aussi confirmé l'importance du soutien de l'entreprise pour hausser la fécondité. Les femmes ayant un travail expriment une plus grande satisfaction à propos de leur vie que celles qui sont à la maison à temps plein, quelle que soit la nature de leur emploi (Bumpass, Rinfuss, Choe et Tsuya, 2009, p. 221; Holloway, 2010, p. 179). On peut donc penser que sans les mesures natalistes mises en place par le gouvernement, l'indice de fécondité, qui a atteint un minimum en 2005, aurait continué à descendre graduellement.

Le principal problème reste que les entreprises ne sont pas pénalisées si elles ne favorisent pas l'application des mesures natalistes, le gouvernement japonais n'étant pas interventionniste (Holloway, 2010, p. 204; Retherford et Ogawa, 2005, p. 33). En 2008, seulement 66,4 \% des entreprises disaient avoir pris des mesures pour faciliter un retrait parental (Ministry of Health, Labour and Welfare of Japan, 2009, p. 12). De plus si, depuis 2005, l'Angel Plan s'applique également aux employés à temps partiel, il est facile pour un employeur d'utiliser de nombreuses clauses spéciales pour rendre inaccessible le congé parental, empêchant de nombreuses femmes de l'utiliser (Lee, Ogawa et Matsukura, 2009, p. 361).

Le journal Asahi Shimbun révélait en 2006, dans une enquête basée sur les statistiques gouvernementales, que plus de $42 \%$ des travailleurs effectuaient des heures supplémentaires non payées. Parmi ces travailleurs, on comptait une forte proportion de jeunes papas, puisque $80 \%$ des pères font plus de 20 heures supplémentaires par mois. Une autre étude confirme que $23 \%$ des pères dans la trentaine travaillent plus de quatre heures supplémentaires par jour, ce qui leur fait des semaines de 60 heures, sans compter le temps de transport (Retherford et Ogawa, 2005, p. 35). La situation économique ne semble pas améliorer les choses, puisqu'entre 2001 et 2005 , la moyenne des heures quotidiennes est passée de 9,2 heures à 10,2 heures, mais le salaire annuel d'un salaryman a pourtant diminué, passant de 6450000 yens en 2001 à 6350000 yens en 2005.

Dans les entreprises où le congé parental est disponible, tout n'est pas rose. Les patrons n'hésitent pas à faire de la pression, particulièrement sur leurs employées, pour qu'elles démissionnent quand elles deviennent trop " âgées ", c'est-à-dire quand elles ont un enfant (Holloway, 2010, p. 187; Jolivet, 1993, p. 69; Yu, 2005, p. 713). Les hommes ne sont pas encouragés à s'engager auprès de leur famille : ces tâches étant considérées comme essentiellement féminines, ils font l'objet de moqueries et de discrimination de la part de leurs collègues et de leurs supérieurs, qui remettent en question leur masculinité (Holloway, 2010, p. 98; Bumpass, Rinfuss, Choe et Tsuya, 2009, p. 221). En 2008, le gouvernement japonais reconnaissait d'ailleurs qu'il était difficile pour les hommes de prendre leur congé parental, quelle que soit la taille de l'entreprise où ils étaient employés (Ministry of Health, Labour and Welfare of Japan, 2009, p. 21). 
Cela sans compter que le Japon est l'un des pays où les parents utilisent très peu la garderie (moins de 20 \% en 2004), alors que les enfants japonais âgés de trois à six ans fréquentent le jardin d'enfants (yochien) à plus de $80 \%$, rejoignant ainsi l'ensemble des pays de l'OCDE. II est difficile d'expliquer le faible taux d'utilisation de la garderie (hoikuen), mais les chercheurs avancent quelques explications. D'abord, envoyer son enfant à la garderie n'est pas bien vu par la société japonaise, toujours influencée par la théorie du psychanalyste Doi (Jolivet, 1993, p. 240; Yu, 2005, p. 697). En 1998, le ministère de la Santé, du Travail et du Bien-Être a d'ailleurs consacré son rapport annuel sur la dénatalité à démystifier, à l'aide d'arguments scientifiques, l'idée que les mères devaient être physiquement proches de leur enfant pendant les trois premières années de sa vie (sansaiji shinwa) (Nakatani, 2006, p. 106). Selon un sondage gouvernemental mené en 2004, 45 \% des mères se sentent mal à l'aise de laisser leur enfant aux soins d'une garderie, car elles ont le sentiment de "l'abandonner » (Jolivet, 1993, p. 12; Retherford et Ogawa, 2005, p. 30).

Ensuite, le gouvernement japonais consacre peu de fonds pour soutenir les parents qui auraient besoin de tels services (moins de $0,4 \%$ de son PIB), au contraire des pays nordiques qui surpassent nettement la moyenne de I'OCDE (0,7\% du PIB). En conséquence, la liste d'attente pour accéder aux garderies (hoikuen) est longue, en plus du fait que l'horaire de garde est tout à fait inadapté aux parents travaillant à temps plein (Holloway, 2010, p. 178; Jolivet, 1993, p. 77; Rebick, 2006, p. 88). II est pourtant prouvé que, pour les parents ayant déjà deux enfants, I'utilisation de la garderie est un incitatif pour un troisième (Lee, Ogawa et Matsukura, 2009, p. 364).

Même si $30 \%$ des pères disent vouloir prendre un congé parental, le nouveau régime prévoit plutôt d'encourager au moins $10 \%$ des pères à prendre leur retrait paternel en 2017, au lieu du maigre 2,6 \% de 2011 (Ministry of Health, Labour and Welfare of Japan, 2009). Le célibat chez les hommes augmente, et ils ont pris le nom peu flatteur d' " herbivores ", en opposition aux " carnivores » qui souhaitent " dévorer » les femmes (Morioka, 2013, p. 3). Or pour comprendre la natalité japonaise, il faut nécessairement parler du mariage, car nous avons vu que le nombre de naissances hors mariage était quasi inexistant au Japon. Parmi les hommes de 54 ans, 12,6 \% n'avaient jamais été mariés en 2000 (alors qu'ils n'étaient que 2,1\% en 1975). Les femmes sont dans la même situation : à 54 ans, 5,8 \% avaient toujours été célibataires, une légère hausse depuis 1975, où elles étaient 4,3 \% (Ogawa, Retherford et Matsukura, 2006, p. 26). Cette tendance ne semble pas vouloir s'inverser puisque, selon un sondage du ministère de la Santé, du Travail et du Bien-être (Ministry of Health, Labour and Welfare, 2009), les jeunes femmes sont plus nombreuses que les hommes à déclarer apprécier leur statut de célibataires, affirmant même qu'elles ne prévoient pas de se marier, ni d'avoir d'enfant (White, 2002, p. 39). En parallèle, les exigences d'un emploi de type régulier ne cessent d'augmenter. En bref, la conciliation travail-famille n'est pas simple pour l'homme parce que le père n'est pas encouragé par l'entreprise à assumer plus de responsabilités domestiques et familiales.

\section{Les impacts pour la famille}

Certaines études ont avancé que, pour qu'un père fasse usage d'un congé paternel, deux conditions doivent être remplies : que le salaire soit assuré à plus de 50 \% et que le congé excède 14 jours (O’Brien, 2009, p. 190). Dans le cas contraire, on assiste plutôt à une augmentation du nombre d'heures de travail des pères, afin de combler la perte du salaire féminin. Ainsi, les mesures prises par l'Islande dépassent par exemple les minimums requis pour inciter les pères à prendre leur congé paternel, en octroyant trois mois de congé à 80 \% du revenu (Harvey, 2014, p. 94).

Ce type de politiques familiales permet la création d'emplois (multipliant les services de garde d'enfants) et encouragent le retour au travail des mères, ce qui permet de contrecarrer la perte de main-d'œuvre due au vieillissement des travailleurs (Jenson, 2008, p. 389). Ces mesures permettent aux parents de passer les premiers mois avec leur enfant, mais l'État s'en sert aussi comme d'un « [...] instrument de conciliation travail-famille, avec pour objectif d'accroître l'employabilité des parents et donc d'assurer la sécurité des revenus » (Jenson, 2008, p. 387). Les congés parentaux s'ajoutent aux ajustements qu'ont dû faire les États et les entreprises pour conserver leurs travailleurs, car on parle de plus en plus d'articulation travail-famille pour encourager les couples qui jugent le travail incompatible avec la venue d’un enfant (Thévenon, 2006, p. 16). Au Japon, les congés parentaux, tout 
comme les différentes mesures de conciliation travail-famille, font donc partie d'un système global visant à encourager la natalité.

Les études qui se sont penchées sur les impacts de la présence du père près de son nouveau-né sont encore peu nombreuses, mais il semble que les conséquences soient positives pour la division des tâches à la maison. En effet, plus la présence du père est prolongée au début de la vie de l'enfant, plus le partage des tâches sera équitable ensuite (Tanaka et Waldfogel, 2007, p. 420). D’après une étude suédoise, plus le congé paternel est long, plus l'implication du père au foyer sera importante, et on a aussi observé une diminution de ses heures de travail par la suite (Haas et Hwang, 2008). Les parents islandais de 2003 rapportent un meilleur partage que ceux de 1997 et la participation des pères aux tâches a augmenté, passant de 30 \% en 2005 à 40,4 \% en 2010 (Gíslasson, 2011, p. 11).

De plus, un enfant ayant bénéficié d'un contact privilégié avec son père dès la petite enfance aurait de meilleurs résultats scolaires (Brandth et Gíslasson, 2011, p. 127). Une récente étude longitudinale faite en Angleterre mentionne également que les bébés, surtout ceux de sexe masculin, ayant souvent interagi avec leur père à l'âge de trois mois auront moins de problèmes de comportement à l'âge d'un an (Ramchandani, Domoney, Sethna, Psychogiou, Vlachos et Murray, 2012, p. 7). Pour les chercheurs, ces résultats indiquent que l'implication d'un père auprès de son bébé est une mesure "préventive " efficace pour contrer de futurs problèmes comportementaux (Ramchandani et collab., 2012, p. 9). D’ailleurs, un meilleur équilibre entre les parents aurait des conséquences à long terme sur la perception par l'enfant des rôles sexués (Gíslasson, 2007, p. 5), ce que viennent confirmer les différentes études de Lamb, qui souligne que les enfants ayant eu des parents avec un partage des tâches plus égalitaire ont moins tendance à attribuer des rôles stéréotypés aux femmes et aux hommes. Finalement, le chercheur souligne que, pour le bébé, il est important de s'attacher à deux personnes, peu importe leur sexe, car la diversité des stimulations est bénéfique à son développement (Lamb, 2010, p. 7).

Pratiquement tous les livres et les articles scientifiques consacrés à la dénatalité japonaise parlent de la division des tâches domestiques. Même le gouvernement du Japon en fait mention dans un document officiel de 2009 intitulé Introduction to the Revised Child Care and Family Care Leave Law, où on souligne que les pères sont beaucoup moins impliqués aux niveaux familial et domestique que les mères. En fait, depuis 2002 et le plan Measures to Cope with a Fewer Number of Children Plus One, l'État japonais pointe du doigt le rôle non assumé du père comme une raison importante du déclin de la fécondité au Japon, et d'autres études le confirment (Retherford et Ogawa, 2005, p. 32). Un rapport faisant la révision des mesures natalistes publié en 2009 y revient : on présente l'anxiété liée à un mauvais partage des tâches au sein du couple comme un frein à la fécondité (Ministry of Health, Labour and Welfare of Japan, 2009, p. 8; OCDE, 2007, p. 13).

On pourrait penser que le peu d'implication des pères à la maison est lié à leur charge de travail excessive, d'autant plus que le rapport de l'OCDE mentionne que le nombre d'heures de travail a tendance à augmenter pour les nouveaux pères (OCDE, 2007, p. 15). Les heures supplémentaires ne sont pas toujours des moments passés au bureau devant l'ordinateur, mais peuvent également être des "moments de socialisation obligatoires " entre collègues. C'est souvent dans ces réunions informelles après le travail que les vraies décisions sont prises. L'entreprise ne s'attend pas à ce qu'un homme doive rentrer à la maison pour être avec sa famille puisque s'il a des enfants, sa femme peut s'en occuper (Yoda, 2000, p. 891).

Comment expliquer qu'un État ayant besoin de stimuler sa natalité n'arrive pas à instaurer des politiques familiales qui fonctionnent ailleurs? Par exemple, est né pour la première fois en Suède l'idée d'un congé uniquement dédié aux hommes : la daddy period. La Norvège l'a instauré en 1993, la Suède en 1995 et le Danemark en 1999. Les changements furent notables : les pères norvégiens n'étaient que $2 \%$ à prendre une part du congé parental avant l'instauration de la mesure, alors qu'en 2000, $85 \%$ des pères prenaient le congé paternel de quatre semaines (O’Brien, 2004, p. 133). Au Québec, le Régime québécois d'assurance parentale, entré en vigueur en 2006, rappelle les politiques familiales des pays scandinaves, et cela a changé la dynamique : " En effet, au Québec, $80 \%$ des pères admissibles au congé de paternité l'utilisent aujourd'hui, comparativement à environ $20 \%$ qui ont utilisé le congé parental canadien au cours de la période 2001-2004 » (Tremblay, 2012, p. 13). 


\section{Conclusion}

Dans cet article, nous avons d'abord exposé les éléments essentiels de la théorie de Beck, notamment sur la société ultramobile de célibataires, et les éléments concernant la société actuelle. Nous avons notamment rappelé que « la répartition des rôles en fonction de l'appartenance sexuelle est la base de la société industrielle [...]. La société industrielle est donc dépendante des situations inégales des hommes et des femmes. " (Beck, 2001, p. 235) Nous avons identifié les trois grands modèles de relation travail-famille, puis situé le Japon dans le modèle non interventionniste, ce qui permet d'autant mieux pourquoi les pères japonais se situent dans le cadre d'une société ultramobiles de célibataires au sens de la théorie de Beck.

Nous avons présenté un ensemble de statistiques qui permettent de confirmer que les femmes japonaises sont les principales responsables de la gestion de la famille et de la résidence, et ce, même si elles travaillent à plein temps. Le partage des tâches est très inégal au Japon, et les femmes ont de la difficulté à retourner travailler après avoir eu un enfant. Un fort pourcentage de femmes continuant à travailler le fait à temps partiel. Nous avons aussi indiqué que les règles de la fiscalité n'encouragent pas non plus le travail à plein temps des femmes, puisque le système d'imposition favorise les femmes gagnant moins de 10000 dollars canadiens par année.

On observe de la part des pères des signes de changement. Mais il n'est pas facile pour un père qui le décide de prendre soin de ses enfants. Les collègues de travail qui en rajoutent, avec leurs remarques moqueuses lorsqu'on invoque des motifs familiaux pour quitter plus tôt les « 5 à 7 » obligatoires qui se terminent tard en soirée. On peut en venir à douter de sa masculinité, à cause de son implication dans un monde pratiquement exclusivement féminin (Rebick et Takenaka, 2006, p. 13). Rien n'est adapté pour le père : les poussettes ont des barres trop courtes pour la taille en moyenne plus élevée des hommes et il ne peut aller changer son enfant dans les toilettes puisqu'il n'y a aucune table à langer (Nakatani, 2006, p. 103). À ces aspects pratiques s'ajoute souvent le sentiment de n'être pas à sa place : aux rencontres scolaires, il est le seul père présent (Jolivet, 1993, p. 95); lorsqu'il se rend à I'hôpital, il peut être questionné sur l'absence de la mère, théoriquement responsable du soin de l'enfant (Nakatani, 2006, p. 103); lorsqu'il se rend à la garderie, on s'interroge : mais pourquoi ce père a le temps de venir chercher son enfant? Serait-il sans emploi? Ces répliques rapportées par les pères montrent deux choses : que les pères qui prennent soin de leurs enfants vont à contre-courant, mais aussi qu'ils existent, de par ces témoignages ${ }^{3}$. Petit à petit, ces papas non conventionnels réussiront peut-être à élargir le rôle du père japonais.

Le modèle de Beck a toutefois ses limites, car il suppose que l'État a le pouvoir de mettre en place des politiques publiques pour soutenir la parentalité. Dans le contexte d’hypermodernité néolibérale (capitalisme, faible interventionnisme de l'État, consommation de masse, haute technologie, coûts élevés de l'éducation et des soins de santé) vécu par la société japonaise, l’État ne dispose pas de toute la latitude nécessaire pour implanter diverses mesures de soutien, comme le recommande Beck. Et c'est un paradoxe important, car le Japon a besoin de toute la main-d'œuvre disponible pour maintenir la croissance de son économie et contrer le vieillissement de sa population. Or, si le mode de production capitaliste est bien ancré en ce qui a trait au travail, l'habitus culturel traditionnel japonais est toujours en place. Ainsi, le Japon offre peu de mesures de conciliation travail-famille, n'incitant tout simplement pas les femmes à être présentes sur le marché du travail, du moins pas à plein temps. Le pays se prive alors d'une main-d'œuvre qualifiée lorsque ces mères occupent un emploi à temps partiel afin d'avoir plus de souplesse dans leurs conditions de travail. II y a donc une profonde contradiction entre les orientations plus traditionnelles et familiales de la société et les réalités socioéconomiques du Japon. Si l'on a modernisé les dispositions relatives au congé des pères et mères, les hommes sont peu enclins à utiliser le congé parental sans solde et les femmes ont subi davantage de discrimination à la suite de l'adoption de la nouvelle loi, puisque les entreprises prévoient qu'elles s'absenteront pour maternité. Ainsi, l'absence de politiques publiques efficaces de soutien à la parentalité contribue bien à faire du Japon une société ultramobile de célibataires, ou du moins à se rapprocher fortement de cette vision.

\footnotetext{
${ }^{3}$ En octobre 2014, le manga Cooking Papa a fêté ses 30 ans d'existence avec son $1300^{\mathrm{e}}$ chapitre. La série dépeint un père qui cuisine pour sa famille et ses collègues, mais qui en vient aussi graduellement à s'impliquer davantage dans les autres tâches de la maison et dans les soins à ses deux enfants.
} 


\section{Références bibliographiques}

Beck, U. (1998). Le conflit des deux modernités et la question de la disparition des solidarités, Lien social et Politiques, 39, 15-25.

Beck, U. (2001). La société du risque. Sur la voie d'une autre modernité. Paris : Champs. (Ouvrage original publié en 1986 sous le titre Risikogesellschaft, Francfort, Suhrkamp).

Beck, U. et Lau, C. (2005). Second modernity as a research agenda: theoretical and empirical explorations in the "meta-change" of modern society, The British Journal of Sociology, 56(4), 525-557.

Bernier, B. (2009). Le Japon au travail, Montréal : Presses de l'Université de Montréal.

Braibant, S. (2014). Les pères japonais paternels victimes de harcèlement au travail. Récupéré le 30 janvier 2015 du site TV5Monde : http://information.tv5monde.com/terriennes/les-peres-japonais-paternels-victimes-deharcelement-au-travail-3160

Brandth, B. et Gíslasson, I. (2011). Family policies and the best interest of children. Dans Parental leave, childcare and gender equality in the Nordic countries (p. 109-145). Copenhague : TemaNord.

Bumpass, L., Rinfuss, R., Choe, M. et Tsuya, N. (2009). The institutional context of low fertility, Asian Population Studies, 5(3), 215-235.

Crompton, S. et Keown, L. (2009). Les prestations parentales influencent-elles les décisions en matière de fécondité? Tendances sociales canadiennes, Statistique Canada, 88, 49-57.

Gíslasson, I. V. (2007). Parental leave in Iceland, bringing the fathers in. Developments in the wake of new legislation in 2000, Akureyri, Islande : Jafnréttisstofa.

Gíslasson, I. V. (2011). Changing Fathers - Reluctant Mothers?, International Network on Leave Policies and Research, Reykjavík, Islande : Háskoli Íslands.

Haas, L. et Hwang, P. (2008). The impact of taking parental leave on fathers' participation in childcare and ties with children: Lessons from Sweden, Community, Work and Family, 11, 85-104.

Hantrais, L. et Letablier, M.-T. (1995). La relation famille-emploi : une comparaison des modes d'ajustement en Europe. Paris : Centre d'études de l'emploi.

Hantrais, L. et Letablier, M.-T. (1996). Familles, travail et politiques familiales en Europe. Paris : Presses universitaires de France.

Harvey, V. (2014). L’homme au foyer avec bébé. Les congés paternels en Islande, Aspects sociologiques, 21(1), 85110.

Hirayama, Y. et Izuhara, M. (2008). Women and housing assets in the context of Japan's home-owning democracy, Journal of Social Politics, 37(4), 641-660.

Holloway, S. (2010). Women and family in contemporary Japan. New York, NY : Cambridge University Press.

Jenson, J. (2008). L'avenir de la famille québécoise. Quel rôle pour les politiques sociales et familiales? Dans G. Pronovost, C. Dumont et I. Bitaudeau (dir.), La famille à l'horizon 2020 (p. 379-400). Québec : Presses de I'Université du Québec.

Jolivet, M. (1993). Un pays en mal d'enfants, crise de la maternité au Japon. Paris : La Découverte.

Kaufmann, J. (2009). L'étrange histoire de l'amour heureux. Paris : Pluriel.

Keizai Koho Center (2010). Japan, an international comparison. Tokyo : Japan Institute for Social and Economic Affairs.

Lamb, M. (2010). How do fathers influence children's development? Let me count the ways. Dans M. Lamb (dir.), The role of the father in child development (p. 1-26). Cambridge, MA : Wiley. 
Lee, S., Ogawa, N. et Matsukura, R. (2009). Is childcare leave effective in raising fertility in Japan? Asian Population Studies, 5(3), 349-369.

Lesemann, F. et Nicol, R. (1994). Politiques familiales : comparaisons internationales. Dans M. Baker (dir.), Les politiques gouvernementales face aux familles canadiennes en transition (p. 131-140). Ottawa : Institut Vanier de la famille.

Matsukura, R., Ogawa, N. et Clark, R. (2007). Analysis of employment patterns and the changing demographic structure of Japan, The Japanese Economy, 34(1), 82-153.

Ministry of Health, Labour and Welfare of Japan (2009). Introduction to the revised Child Care and Family Care Leave Law. http://www.mhlw.go.jp/english/policy/affairs/dl/05.pdf

Morioka, M. (2013). A phenomelogical study of "Herbivore Men". The Review of Life Studies, 4, 1-20.

Moss, P. (2010). International Review of Leave Policies and Related Research 2010. London, R.-U. : Department of Trade and Industry. Récupéré le 27 février 2011 de :

http://www.leavenetwork.org/lp_and_r_reports/country_notes/?type=98

Moss, P. et Kamerman, S. (dir.) (2009). The politics of parental leave policies. Children, parenting, gender and the labour market. Bristol, R.-U. : The Policy Press.

Nakatani, A. (2006). The emergence of "nurturing fathers": Discourses and practices of fatherhood in contemporary Japan. Dans M. Rebick et A. Takenaka (dir.), The changing Japanese family (p. 94-108). New York, NY : Routledge.

Nations Unies (2011). Men in families and family policy in a changing world. New York, NY : Nations unies.

O'Brien, M. (2004). Social science and public policy perspectives on fatherhood in the European Union. Dans M. Lamb (dir.), The role of the father in child development (p. 121-145). Cambridge, MA : Wiley.

O'Brien, M. (2009). Fathers, parental leave policies, and infant quality of life: International perspectives and policy impact. The Annals of the American Academy of Political and Social Science, 624, 190-213.

O'Brien, M. (2011). Report on United Nations International Year of the Family 2014 Preparations. High North Extremely Different?: Leave policies within the family-related policies. Reykjavík, Islande $: 8^{\text {th }}$ seminar of International Network on Leave Policies and Research.

OCDE (2007). Bébés et employeurs: comment réconcilier travail et vie de famille, Synthèse des résultats dans les pays de l'OCDE. Paris : OCDE.

Ogawa, N., Retherford, R. D. et Matsukura, R. (2006). Demographics of the Japanese family. Dans M. Rebick et A. Takenaka (dir.), The changing Japanese family (p. 19-38). New York, NY : Routledge.

Parsons, T. (1964). The father symbol: An appraisal in the light of psychoanalytic and sociological theory. Dans T. Pearson, Social Structure and Personality (chapitre 2). Londres, R.-U. : Collier-MacMillan.

Powell, M. et Barrientos, A. (2004). Welfare regimes and welfare mix. European Journal of Political Research, 43, 85-105.

Rebick, M. (2006). Changes in the workplace and their impact on the family. Dans M. Rebick et A. Takenaka (dir.), The changing Japanese family (p. 3-16). New York, NY : Routledge.

Rebick, M. et Takenaka, A. (2006). The changing Japanese family. Dans M. Rebick et A. Takenaka (dir.), The changing Japanese family (p. 75-93). New York, NY : Routledge

Retherford, R. D., Ogawa, N. et Matsukura, R. (2001). Late marriage and less marriage in Japan, Population and Development Review, 27(1), 65-102.

Retherford, R. D. et Ogawa, N. (2005). Japan's baby bust: Causes, implications, and policy responses. Dans F. Harris (dir.), The baby bust: who will do the work? Who will pay the taxes? Boulder, $\mathrm{CO}$ : Rowman and Littlefield. 
Ramchandani, P., Domoney, J., Sethna, V., Psychogiou, L., Vlachos, H. et Murray, L. (2012). Do early father-infant interactions predict the onset of externalising behaviours in young children? Findings from a longitudinal cohort study, Journal of Child Psychology and Psychiatry, 19 juillet.

Sakai, J. (2003). Shoushi (Dénatalité). Tokyo : Kodansha.

Tanaka, S. et Waldfogel, J. (2007). Effects of Parental Leave and Work Hours on Fathers' Involvement with their Babies, Community, Work and Family, 10(4), 409-426.

Thévenon, O. (2006). What are the Challenges for Social Welfare Reforms in Continental and Southern Europe? Revue française des Affaires sociales, 5, 5-18.

Tremblay, D. (2012). Articuler emploi et famille. Le rôle du soutien organisationnel au cœur de trois professions, Québec : Presses de l'Université du Québec.

White, M. (2002). Perfectly Japanese: making families in an era of upheaval, Berkeley et Los Angeles, CA : University of California Press.

Yoda, T. (2000). The rise and fall of maternal society: Gender, labor and capital in contemporary Japan, South Atlantic Quarterly, 99(4), 865-902.

Yu, W. (2005). Changes in women's postmarital employment in Japan and Taiwan, Demography, 42(4), 693-717. 